\title{
O pêndulo invertido sob diferentes perspectivas
}

\author{
The inverted pendulum under different perspectives \\ Thiago de Cacio Luchese*1@ \\ ${ }^{1}$ Universidade Federal da Fronteira Sul, Cerro Largo, RS, Brasil
}

Recebido em 21 de Dezembro, 2017. Revisado em 20 de Março, 2018. Aceito em 03 de Abril, 2018.

\begin{abstract}
Três diferentes explanações sobre o fenômeno da oscilação invertida de um pêndulo com pivô verticalmente forçado são apresentadas. Duas destas abordagens estão descritas na literatura e uma compilação de resultados é apresentada, enquanto que a terceira visa estimular estudantes a associarem ferramentas matemáticas com a descrição do fenômeno físico observado, em uma busca de quantificá-lo, mesmo que de modo aproximado. A possibilidade de separação em distintas escalas de tempo de um único fenômeno factível em sala de aula torna-se muito nítida, sendo a utilidade e as limitações desse tipo de descrição temporalmente escalonada explicitadas. Ao término da apresentação das diferentes explanações alguns resultados numéricos são comparados com a terceira abordagem, trazendo a realidade atual de investigação científica: as simulações computacionais. Trata-se de uma oportunidade ímpar para estudantes terem contato com ferramentas matemáticas diversas tendo a intuição física guiando suas aplicações.
\end{abstract}

Palavras-chave: equação de Mathieu, potencial médio, série aproximativa, escala temporal.

Three different explanations for the phenomenon of inverted oscillation of a pendulum with vertically forced pivot are presented. Two of these approaches are present in the literature and a compilation of results have been presented, while a third one aims to stimulate students to associate mathematical tools with the description of the observed physical phenomenon, searching for a quantification, even approximated. The possibility of separating into different time scales of a single phenomenon feasible in the classroom becomes very clear, and the utility and limitations of this type of description are exposed. At the end of the presentation of the different explanations some numerical results are compared with the third approach, bringing the current reality of scientific research: computational simulations. It is an unparalleled opportunity for students to come in contact with diverse mathematical tools with physical intuition guiding their applications.

Keywords: Mathieu equation, average potential, aproximating series, temporal scales.

\section{Introdução}

Uma barra vertical suspensa em uma de suas extremidades com a possibilidade de girar em torno do ponto de suspensão constitui um pêndulo físico, sendo o ponto de suspensão denominado pivô. Quando a barra assim suspensa é perturbada de sua posição de equilíbrio inicia-se um processo de oscilação amortecida até que ela, após um certo intevalo de tempo, retorne novamente à posição inicial de repouso.

Embora a oscilação acima descrita seja comum e o pêndulo físico se constuitua em um problema de estudo padrão no ensino de Física, pouco se fala sobre o fato de que um forçamento periódico do pivô na direção vertical pode fazer com que o pêndulo físico venha a se equilibrar na posição invertida. Dito de outra maneira, a oscilação periódica do pivô na direção vertical pode, sob certas condições, gerar mais um ponto de equilíbrio estável para o pêndulo físico, no qual o amortecimento da oscilação o fará parar de oscilar quando seu centro de massa ficar acima do pivô oscilante.

*Endereço de correspondência: thiago.luchese@uffs.edu.br
O problema da inversão de um pêndulo por meio da excitação de seu pivô já foi largamente estudado [1]- 8], porém pouco divulgado no âmbito de revistas de livre acesso no idioma português. Pretende-se que este trabalho seja de valia no sentido de divulgar e incentivar o estudo deste sistema simples e de fácil construção que permite abordar temas tão complexos quanto a aproximação de regimes dinâmicos por separação de escalas de tempo características 2, 3], tal qual acontece nas descrições de modos ro-vibracionais de moléculas 9 .

Desse modo a obtenção do intervalo de parâmetros de forçamento do pivô que levam o pêndulo a estabilizar-se na posição invertida fez-se objeto de estudo deste trabalho, na intenção de aproveitar um sistema simples para abordar diferentes técnicas físico-matemáticas. Uma oscilação senoidal foi imposta ao pivô, com amplitude e frequência conhecidas, e então algumas abordagens analíticas para o regime de pequenas oscilações do pêndulo no entorno da posição invertida foram construídas.

No que segue, primeiramente formaliza-se o problema com a obtenção da equação de movimento do pêndulo com pivô forçado verticalmente. Então a solução dessa 
equação é abordada seguindo as referências [1] e [2]. O uso destas referências como base de comparação de resultados a serem aqui apresentados justifica-se pela forma didática com que o problema é por elas tratado. $\mathrm{Na}$ referência [1] encontra-se o rigor matemático sob uma descrição clara e reprodutível (com algum esforço) que, embora forneça uma forma de trabalho deslocada da física do problema e que dificilmente poderia ser abordada em um curso regular de graduação, possui como benefício ser um exemplo de exposição de um assunto complexo de maneira muito objetiva. Já na referência [2] a física do problema é invocada no decorrer de toda a resolução e as aproximações são usadas de maneira bastante intuitiva, explicitando-se as motivações que levam a adotá-las, tornando a matemática envolvida extremamente simples.

Em seguida a este resgate de resultados da literatura ao presente objeto de estudo, dá-se uma abordagem analítica diferenciada ao pêndulo invertido e que tem a pretensão de misturar algumas das qualidades observadas nas referências Phelps-Hunter [1] e Butikov [2]. Tal abordagem baseou-se na referência [10, onde o uso de ferramentas matemáticas básicas com uma metodologia aproximativa não desvinculada da física pretende gerar reflexões a respeito da forma como fatos experimentais podem guiar a obtenção da solução de problemas levemente sofisticados, mesmo que estas soluções sejam aproximadas e limitadas. Com esta terceira abordagem ao mesmo problema, espera-se construir a confiança de que abordagens limitadas não são sinônimos de incapacidade de construção de resultados quantitativamente corretos, sensação esta muito presente no início da graduação em física, onde "tudo é aproximado".

Ao término do trabalho alguns resultados de simulações numéricas são comparados com as previsões da terceira das abordagens dadas ao pêndulo invertido no que diz respeito às combinações dos parâmetros amplitude e frequência capazes de estabilizar a posição invertida, tecendo-se em seguida as considerações finais.

\section{Revisando resultados}

\subsection{A equação de movimento}

Seja um pêndulo físico constituído por uma barra de comprimeto $L$ e massa $m$, presa em uma de suas extremidades a um pivô que se desloca na direção vertical segundo a função

$$
y_{0}(t)=a \cos (\omega t)
$$

onde $y_{0}$ designará, a partir de agora, a posição do pivô em um sistema coordenado com eixo $x$ horizontal, eixo $y$ vertical e origem no ponto médio de oscilação do pivô, conforme ilustrado na Figura 1. Serão investigadas as condições sobre os parâmetros de forçamento do pivô, a saber a amplitude $a$ e a frequência $\omega$, capazes de produzir a estabilidade invertida do pêndulo.

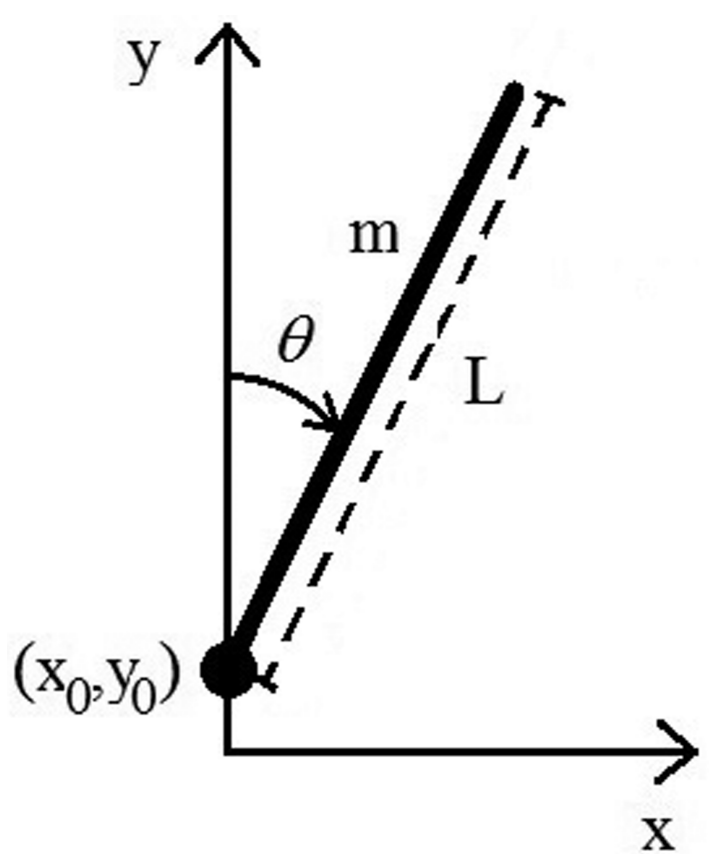

Figura 1: Sistema de coordenadas e grandezas importantes para estabelecimento do problema em estudo.

A equação de movimento será obtida a partir da Lagrangiana do sistema. Sendo assim, segue-se a obtenção das energias cinética e potencial como função do tempo e da posição do pêndulo.

A energia cinética é dada por:

$$
T=\frac{1}{2} m\left(\dot{x}^{2}+\dot{y}^{2}\right)+\frac{1}{2} I_{0} \dot{\theta}^{2}
$$

sendo $(x, y)$ as coordenadas do centro de massa da barra, $\theta$ o ângulo de deflexão da barra, contado no sentido horário a partir do eixo $y$ positivo, e $I_{0}$ o momento de inércia em relação ao centro de massa. Está sendo usada a notação $\dot{\beta}$ para indicar a derivada temporal da variável $\beta$. Seja notado também que a rigidez da barra e a fixação de um de seus extremos no pivô impõem que os ângulos de rotação em torno de seu centro de massa e de rotação em torno do pivô sejam idênticos, o que permite expressar a energia cinética de rotação conforme indicado na equação (2).

As coordenadas $(x, y)$ do centro de massa da barra estão relacionadas ao ângulo de deflexão $\theta$ por meio das expressões:

$$
\begin{aligned}
& x=l \operatorname{sen} \theta \\
& y=y_{0}+l \cos \theta
\end{aligned}
$$

em que $l(=L / 2)$ é a distância do centro de massa da barra até o pivô. Com isso a energia cinética total pode ser escrita como função do ângulo $\theta$

$$
T=\frac{1}{2} m \dot{y}_{0}^{2}+\frac{1}{2} I \dot{\theta}^{2}-m l \dot{\theta} \dot{y}_{0} \operatorname{sen} \theta
$$

onde $I=m l^{2}+I_{0}$ é o momento de inércia do pêndulo em relação ao pivô. 
Por sua vez, a energia potencial é dada por:

$$
V=m g y=m g y_{0}+m g l \cos \theta .
$$

De posse das expressões para as energias cinética e potencial, escreve-se a Lagrangiana $(\mathcal{L}=T-V)$ do sistema conforme segue

$$
\mathcal{L}=\frac{1}{2} m \dot{y}_{0}^{2}+\frac{1}{2} I \dot{\theta}^{2}-m l \dot{\theta} \dot{y}_{0} \operatorname{sen} \theta-m g y_{0}-m g l \cos \theta
$$

e a equação de movimento resultante da aplicação da relação de Euler-Lagrange $\frac{d}{d t} \frac{\partial \mathcal{L}}{\partial \dot{\theta}}-\frac{\partial \mathcal{L}}{\partial \theta}=0$, após rearranjo, fica dada por

$$
\left(\frac{I}{m l}\right) \ddot{\theta}-\left(\ddot{y}_{0}+g\right) \operatorname{sen} \theta=0 .
$$

Uma vez que a posição do pivô é dada pela equação (1), pode-se reescrever a equação de movimento acima sob a seguinte forma

$$
\ddot{\theta}+\left(-\omega_{0}^{2}+\frac{a l m \omega^{2}}{I} \cos \omega t\right) \operatorname{sen} \theta=0
$$

onde foi definida a grandeza

$$
\omega_{0}^{2} \equiv \frac{m g l}{I}
$$

que corresponde à frequência natural do pêndulo em sua posição ordinária. (Notar que esta equação de movimento não se reduz ao caso do pêndulo simples quando a amplitude de oscilação do pivô é igualada a zero. Isso ocorre porque o ângulo de deflexão do pêndulo foi tomado a partir da direção vertical para cima.)

\subsection{Resultados da abordagem dada por Phelps-Hunter 1]}

Para pequenos desvios da posição vertical invertida temse $\theta \ll 1$ e vale a aproximação $\operatorname{sen} \theta \cong \theta$. Nesse caso a equação de movimento (3) é reescrita como

$$
\ddot{\theta}+\left(-\omega_{0}^{2}+\frac{a l m \omega^{2}}{I} \cos \omega t\right) \theta=0 .
$$

Uma troca de variáveis nesta equação permite reconhecêla como a equação de Mathieu, uma equação diferencial bastante estudada e aplicável em diferentes contextos [10]. A troca a ser proposta consiste em tornar o tempo adimensional por meio da definição da nova variável $\tau$

$$
\tau \equiv \frac{\omega}{2} t
$$

e das constantes auxiliares, também adimensionais, $\theta_{0}$ e $\theta_{1}$

$$
\begin{aligned}
\theta_{0} & \equiv-4 \frac{\omega_{0}^{2}}{\omega^{2}} \\
\theta_{1} & \equiv \frac{2 a l m}{I}
\end{aligned}
$$

A substituição destas definições na equação (4) permite reexpressá-la conforme segue

$$
\frac{d^{2} \theta}{d \tau^{2}}+\left(\theta_{0}+2 \theta_{1} \cos 2 \tau\right) \theta=0
$$

cuja solução pode ser construída a partir da seguinte representação em série

$$
\theta(\tau)=e^{i \lambda \tau} \sum_{n=-\infty}^{\infty} b_{n} e^{2 i n \tau} .
$$

Quando esta série é substituída na equação (7), um rearranjo de termos e o uso da identidade $2 \cos 2 \tau=$ $e^{2 i \tau}+e^{-2 i \tau}$ permite reescrevê-la conforme segue

$$
\begin{gathered}
\sum_{n=-\infty}^{\infty}\left\{\theta_{1} b_{n-1}+\left[\theta_{0}-(\lambda+2 n)^{2}\right] b_{n}+\right. \\
\left.\theta_{1} b_{n+1}\right\} e^{i(2 n+\lambda) \tau}=0 .
\end{gathered}
$$

A equação 9 só será satisfeita para um $\tau$ arbitrário se cada um dos coeficientes for identicamente nulo. Desse modo é obtida a seguinte relação de recorrência entre os coeficientes $b_{n}$ 's

$$
\theta_{1} b_{n-1}+C_{n} b_{n}+\theta_{1} b_{n+1}=0
$$

tendo sido definida a constante auxiliar $C_{n}$

$$
C_{n} \equiv \theta_{0}-(2 n+\lambda)^{2}
$$

lembrando que $n$ assume qualquer valor inteiro, positivo ou negativo.

Uma forma de determinar os coeficientes $b_{n}$ em aproximações sucessivamente mais precisas é obtida pela separação da equação de recorrência 10 em dois grupos, os que possuem $n>0$ e os que possuem $n<0$. A rápida convergência da série permite assumir que após um dado $|n| \geq N$, sendo $N$ um valor máximo de $|n|$ que depende do grau de precisão desejado na determinação dos coeficientes $b_{n}$, pode-se desprezar o termo $b_{n+1}$ na recorrência (10) frente ao $b_{n}$ e $b_{n-1}$. Essa hipótese leva às seguintes representações dos coeficientes $b_{n},|n|<N$, na forma de frações continuadas

$$
\begin{aligned}
& b_{n}=-\frac{\theta_{1} b_{n-1}}{C_{n}-\frac{\theta_{1}^{2}}{C_{n+1}-\frac{\theta_{1}^{2}}{C_{n+2}-\cdots}}} ; n>0 \\
& b_{n}=-\frac{\theta_{1} b_{n+1}}{C_{n}-\frac{\theta_{1}^{2}}{C_{n-1}-\frac{\theta_{1}^{2}}{C_{n-2}-\cdots}}} ; n<0
\end{aligned}
$$

Note-se que, sendo cada fração continuada de recorrência regressiva, ambas dependerão da determinação do coeficiente $b_{0}$ que, por sua vez, ficará arbitrário para ajuste de condições iniciais. Esse fato pode ser verificado quando tenta-se substituir $b_{1}$ e $b_{-1}$, conforme frações acima indicadas, na equação 10 com $n=0$. Como consequência do cancelamento do coeficiente $b_{0}$ na equação 
(10) resta um polinômio a ser resolvido em $\lambda$, o que, por sua vez, fornecerá este último valor a ser determinado para completar a solução. Escolhe-se $0 \leq \lambda \leq 1$, caso exista solução real.

Notando que em uma primeira aproximação o polinômio a ser resolvido em $\lambda$ é de ordem 6 , cabe explicitar que Phelps-Hunter [1] também trazem o seguinte resultado para obtenção de $\lambda$, após um certo trabalho algébrico sob as aproximações $\theta_{0} \ll 1$ e $\theta_{1} \ll 1$

$$
\operatorname{sen}^{2} \frac{\pi}{2} \lambda=\frac{\pi^{2}}{4}\left[\frac{2 a^{2} l^{2} m^{2}}{I^{2}}-4 \frac{\omega_{0}^{2}}{\omega^{2}}\right]
$$

equação esta mais simples de ser resolvida que o polinômio oriundo das aproximações dos coeficientes $b_{n}$ e, ainda, fornecendo a possibilidade de continuidade do trabalho analítico a respeito da análise de possibilidade de estabilidade invertida.

Ao término da determinação dos coeficientes $b_{n}$ e $\lambda$, cabe ressaltar que a solução física será somente a parte real da série (8).

A solução em série dada pela equação (8) resultará em um movimento oscilatório somente se o $\lambda$, dado pela equação 11, for real. Essa exigência impõe que

$$
0<\operatorname{sen}^{2} \frac{\pi}{2} \lambda<1
$$

que, por sua vez, usando-se a equação (11) e fazendo-se um rearranjo na desigualdade, implica os limites inferior e superior da amplitude de oscilação $(a)$ em função da frequência de forçamento $(\omega)$, para que haja estabilidade invertida do pêndulo

$$
\sqrt{2} \frac{I}{m l^{2}} \frac{\omega_{0}}{\omega}<\frac{a}{l}<\sqrt{2} \frac{I}{m l^{2}} \frac{\sqrt{\omega^{2}+\pi^{2} \omega_{0}^{2}}}{\pi \omega} .
$$

Seja notado que a série solução, equação (8), quando $\lambda$ é real, corresponde a uma função que oscila lentamente $\left(e^{i \lambda \tau}\right)$ modulando uma outra parte que oscila mais rapidamente $\left(\sum_{n=-\infty}^{\infty} b_{n} e^{2 i n \tau}\right)$. Fisicamente é exatamente isso que acontece: quando o pêndulo oscila na posição invertida há uma "tremulação rápida" da barra, gerada pela excitação do pivô, enquanto ela mesma oscila "lentamente" no entorno daquela posição invertida, sendo o tempo de uma oscilação completa da barra muito maior que o tempo da "tremulação". Essa separação temporal permite quantificar os termos "rápido" e "lento" neste contexto.

A Figura 2 apresenta a evolução temporal (numérica) de $\theta(\tau)$ obtida com o método de Runge-Kutta de quarta ordem aplicado à equação de Mathieu, equação (7), condições iniciais $\theta(0)=0.1$ e $\theta(0)=0$ e parâmetros $\theta_{0}=-2 \times 10^{-3}$ e $\theta_{1}=0.2$. Estes parâmetros correspondem a um pêndulo constituído de uma barra pivotada de comprimento $10 \mathrm{~cm}$, amplitude de oscilação de $6 \mathrm{~mm}$ e frequência de oscilação do pivô $86 \mathrm{~Hz}$. Juntamente com o resultado da simulação (linha sólida) apresenta-se a posição do pivô com o tempo (linha pontilhada preta) e

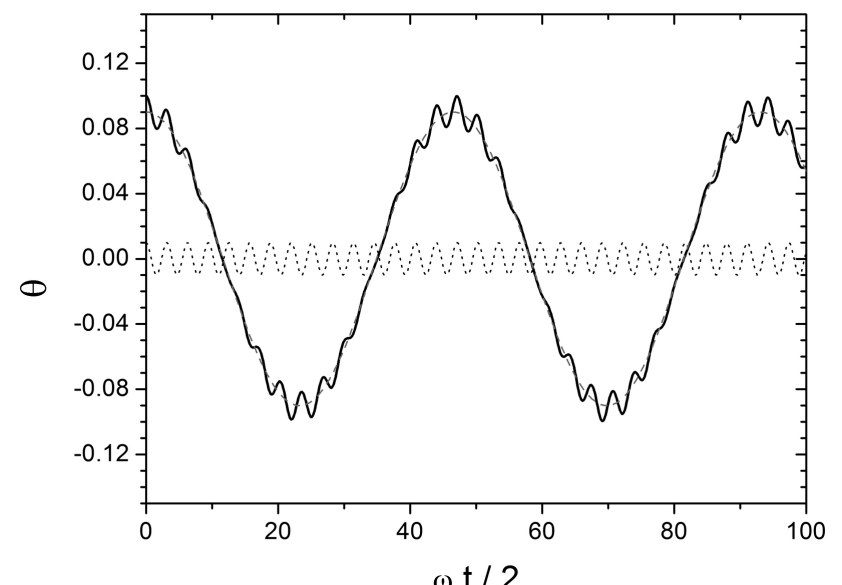

Figura 2: Simulação numérica da oscilação invertida do pêndulo (linha sólida preta) - parâmetros indicados no texto. A oscilação "lenta" (linha tracejada vermelha) é moduladora de uma oscilação "rápida" (linha pontilhada preta), conforme sugerido pela representação em série da solução analítica da equação de Mathieu, equação (8).

uma oscilação puramente senoidal (linha tracejada vermelha), tornando possível visualizar que o movimento completo do pêndulo invertido é, de fato, composto de dois movimentos com escalas temporais distintas.

\subsection{Resultados da abordagem dada por Butikov 2}

Enquanto Phels e Hunter [1] tratam de apresentar a equação de movimento e resolvê-la, Butikov 2] aborda o problema primeiro de um ponto de vista qualitativo para, posteriormente, quantificá-lo, à maneira do que será apresentado a seguir.

Em um dado instante do movimento do pêndulo este formará um ângulo $\theta$ com a vertical e o pivô estará sendo acelerado no sentido do eixo $y$ negativo. Com um pouco de esforço imaginativo, nota-se que tal aceleração irá provocar um decréscimo no módulo do ângulo $\theta$. Caso a aceleração do pivô seja no sentido oposto haverá um acréscimo do módulo deste ângulo. Se a amplitude $a$ de oscilação do pivô for muito menor que a distância $l$ do centro de massa da barra até o pivô $(a \ll l)$ faz-se intuitivo esperar que os desvios ocasionados no ângulo $\theta$, devido a essas acelerações harmônicas do pivô, sejam bem pequenos. Desse modo, o ângulo $\theta$ do pêndulo pode ser decomposto em duas componentes

$$
\theta=\psi+\delta
$$

onde a componente $\psi$ representará a oscilação lenta do pêndulo (lembrando o termo $e^{i \lambda \tau}$ do método anterior) e a componente $\delta$ gerará pequenos desvios no entorno deste $\psi$ (lembrando o somatório).

Ainda em um exercício imaginativo sobre o pêndulo oscilando aos arredores da posição invertida, cabe notar que as deflexões $\delta$ em torno do angulo $\psi$ são tanto 
maiores quanto mais afasta-se da posição de equilíbrio invertida. Não é errôneo expressar matematicamente esta observação sob a seguinte proporção: $\delta \propto \operatorname{sen} \psi$.

Unindo estas informações a respeito do desvio $\delta$ como função de $\psi$ (pequeno e proporcional a sen $\psi$ ) e, ainda, sabendo que ele é gerado exclusivamente pela oscilação do pivô, arbitra-se que sua dependência temporal possa ser escrita como

$$
\delta=-\frac{a}{l} \operatorname{sen} \psi \cos \omega t
$$

cuja validação se dá a posteriori, por meio da comparação dos resultados teóricos decorrentes desta hipótese com resultados de simulações numéricas.

Em um referencial não-inercial fixo ao pivô (neste referencial o pivô permanece fixo), o centro de massa da barra oscilante sofre um torque, doravante denominado torque inercial $\left(\tau_{i}\right)$, com frequência e amplitude diretamente relacionadas à frequência e amplitude de oscilação do referencial (que são propriamente a frequência e amplitude de oscilação do pivô do ponto de vista do referencial inercial em que a análise estava ocorrendo anteriormente). Em um dado tempo $t$, em que o ângulo de deflexão do pêndulo seja $\theta$, este torque inercial será expresso como

$$
\begin{aligned}
\tau_{i} & =\left(-m \ddot{y}_{0}\right) l \operatorname{sen} \theta \\
& =m a l \omega^{2} \operatorname{sen} \theta \cos \omega t .
\end{aligned}
$$

Uma vez que $\theta=\psi+\delta$, tem-se a relação

$$
\operatorname{sen} \theta=\operatorname{sen}(\psi+\delta)=\operatorname{sen} \psi \cos \delta+\operatorname{sen} \delta \cos \psi
$$

e, como $\delta \propto \frac{a}{l}$ sendo $a \ll l$, então conclui-se que $\delta \ll 1$ e valem as aproximações sen $\delta \cong \delta$ e $\cos \delta \cong 1$, gerando o resultado também aproximado para $\theta$

$$
\operatorname{sen} \theta \cong \operatorname{sen} \psi+\delta \cos \psi
$$

Quando este valor para sen $\theta$ é substituído na equação para o torque inercial, equação (14), e usa-se a proposta de dependência temporal do desvio $\delta$ dada pela equação (13), obtém-se

$$
\tau_{i}=\operatorname{mal} \omega^{2} \operatorname{sen} \psi \cos \omega t\left[1-\frac{a}{l} \cos \psi \cos \omega t\right] .
$$

Seja observado nesta expressão para o torque inercial o comportamento variável, ora como torque restaurador (quando $\cos \omega t<0$ ), ora como um que não o é (quando $\cos \omega t>0)$. Apesar disto, em uma média sobre um período de oscilação do pivô é de se esperar que ele seja restaurador pois a posição invertida é observada estável.

Assumindo que $\omega \gg \omega_{0}$, de modo que durante um período de oscilação do pivô o ângulo $\psi$ possa ser considerado constante, obtém-se a seguinte expressão para o torque inercial médio neste períodd ${ }^{1}$

$$
\left\langle\tau_{i}\right\rangle=-\frac{m a^{2} \omega^{2}}{2} \operatorname{sen} \psi \cos \psi
$$

\footnotetext{
${ }^{1}$ Nesta seção os valores médios serão tomados sobre o período de oscilação do pivô $(T=2 \pi / \omega)$, sendo dados pela seguinte expressão: $\langle f\rangle=\frac{1}{T} \int_{t}^{t+T} f\left(t^{\prime}\right) d t^{\prime}$
}

$\mathrm{Ou}$

$$
\left\langle\tau_{i}\right\rangle=-\frac{m a^{2} \omega^{2}}{4} \operatorname{sen} 2 \psi
$$

que é um torque restaurador, conforme esperado (aqui acontece a separação de escalas temporais).

Em contrapartida, o torque gravitacional $\left(\tau_{g}\right)$ em qualquer dos referenciais, inercial ou não, é dado por:

$$
\begin{aligned}
\tau_{g} & =m g l \operatorname{sen} \theta \\
\tau_{g} & \cong m g l \operatorname{sen} \psi-\frac{m g a}{2} \operatorname{sen} 2 \psi \cos \omega t
\end{aligned}
$$

onde foram usadas as expressões 113 e (15). O valor médio deste torque em um período de oscilação do pivô é dado por:

$$
\left\langle\tau_{g}\right\rangle=m g l \operatorname{sen} \psi
$$

Neste ponto fica evidente que o equilíbrio invertido do pêndulo só ocorrerá quando a inequação

$$
\left|\left\langle\tau_{i}\right\rangle\right|>\left|\left\langle\tau_{g}\right\rangle\right|
$$

for satisfeita. Substituindo as expressões obtidas para os torques médios, equações $(16)$ e $(17)$, na inequação $(18)$, é obtida a seguinte amplitude mínima de forçamento para uma dada frequência do pivô, como requisito para produzir a inversão

$$
\frac{a}{l}>\sqrt{2} \sqrt{\frac{I}{m l^{2}}} \frac{\omega_{0}}{\omega} .
$$

É interessante notar que, embora a análise provida por Butikov [2] não seja tão rigorosa quanto aquela dada por Phelps-Hunter [1], o mesmo resultado para o limite inferior de $a$ é obtido caso tenha-se um pêndulo simples com pivô excitado, ou seja, caso $I=m l^{2}$ nas equações (12) e (19).

Observando que nesta abordagem não houve a restrição da dinâmica a pequenas deflexões $\theta$, pode-se obter outro dado valioso desta abordagem: os ângulos de equilíbrio instável $\left(\psi_{M}\right)$, ângulos em que os torques inercial e gravitacional se igualam e o pêndulo pode seguir, arbitrariamente, para sua posição invertida ou ordinária. Tal ângulo é obtido a partir da condição

$$
\left|\left\langle\tau_{i}\right\rangle\right|=\left|\left\langle\tau_{g}\right\rangle\right|
$$

que, quando substituídas as expressões respectivas para $\left\langle\tau_{i}\right\rangle$, equação 16$)$, e $\left\langle\tau_{g}\right\rangle$, equação 17$)$, fornecem o seguinte valor para $\psi_{M}$ :

$$
\psi_{M}=\arccos \left[2 \frac{I}{m l^{2}}\left(\frac{l}{a} \frac{\omega_{0}}{\omega}\right)^{2}\right] .
$$

Resultados numéricos apresentados por Butikov 2] confirmam suas previsões de ângulos de máxima deflexão da posição invertida $\left(\psi_{M}\right)$ e do limite de amplitude de forçamento do pivô capazes de gerar estabilidade invertida para uma dada frequência, validando essa abordagem. 
A análise dos potenciais médios que geram os torques médios inercial e gravitacional é também altamente instrutiva. Ambos os potenciais são obtidos por integração direta (em relação a $\psi$ ) dos respectivos torques médios, uma vez que estes valores médios podem ser considerados advindos de forças conservativas.

O potencial gravitacional médio é

$$
\left\langle U_{g}\right\rangle=m g l \cos \psi
$$

Por sua vez, o potencial inercial médio é

$$
\left\langle U_{i}\right\rangle=-\frac{m a^{2} \omega^{2}}{8} \cos 2 \psi .
$$

O potencial efetivo sob o qual ocorre o movimento pendular corresponde à soma destes dois potenciais. Nesta soma torna-se explícito que a presença do potencial inercial gera um mínimo de pontecial (equilíbrio estável) na posição invertida, em $\psi=0$, caso a inequação (18) seja obedecida. Também evidencia-se que a frequência de oscilação na posição ordinária do pêndulo torna-se mais elevada. E finalmente, os ângulos de máximo potencial efetivo (equilíbrio instável) fornecerão as deflexões de equilíbrio instável $\left(\psi_{M}\right)$.

\section{Uma abordagem diferenciada}

A metodologia a ser seguida a partir de agora para encontrar a solução da equação de movimento difere dos dois métodos abordados anteriormente no sentido de que, conforme se verá, constrói-se uma dada função que satisfaça a equação de movimento e, então, interpretam-se os seus resultados. É uma mistura das duas formas de resolver anteriores: são utilizados argumentos baseados na dinâmica observada do pêndulo invertido para propor soluções aproximadas, aos moldes do efetuado por Butikov [2] e, também, força-se alguns parâmetros de funções a terem determinados valores para que a proposta aproximada satisfaça a equação de movimento, aos moldes de Phelps-Hunter [1]. Com este procedimento (inspirado nas primeiras seções da referência [10], que trata da equação de Mathieu) chega-se a algumas previsões a respeito dos limites dos parâmetros que produzem a estabilidade invertida do pêndulo, limites parcialmente concordantes com os resultados já apresentados.

Conforme já exposto, a equação de movimento (3), após linearização e adimensionalização, pode ser escrita sob a forma apresentada na equação (7), sendo os parâmetros $\theta_{0}$ e $\theta_{1}$ independentes um do outro. No entanto, embora haja essa independência entre $\theta_{0}$ e $\theta_{1}$, deve-se relembrar a pergunta sob investigação neste trabalho: qual(is) o(s) intevalo(s) de parâmetros amplitude e frequência de forçamento do pivô capaz(es) de produzir a estabilidade invertida de um pêndulo? A resposta a esta pergunta, conforme abordagens anteriores, vinculava um intervalo de amplitudes de forçamento a uma dada frequência de forçamento. Notando que a frequência de forçamento está associada à constante $\theta_{0}$ e a amplitude de forçamento à constante $\theta_{1}$, respectivamente equações (5) e (6), pode-se compreender que uma forma de construir uma solução estável é vincular estas constantes, escrevendo uma como função da outra. Sendo assim, arbitra-se que tal dependência seja admitida e expressa sob a seguinte série (a ser justificada posteriormente pela capacidade de previsão de resultados)

$$
\theta_{0}=\nu^{2}+\sum_{n=1}^{\infty} \alpha_{n} \theta_{1}^{n}
$$

de modo que, caso $\theta_{1}$ seja nulo (amplitude de forçamento nula), tem-se $\theta_{0}=\nu^{2}$, o que permite identificar $\nu$ como a frequência natural de oscilação do pêndulo associado à equação (7) quando $\theta_{1}=0$. Os coeficientes $\alpha_{n}$ são constantes a serem determinadas.

A observação experimental de estabilidade do pêndulo invertido serve de motivação para construir uma solução para $\theta(\tau)$ que seja limitada quando $\tau \rightarrow \infty$. Desse modo o aparecimento desse tipo de solução será forçado, propondo-a sob a forma de outras séries no parâmetro $\theta_{1}$

$$
\begin{aligned}
& \theta_{c}(\tau)=\cos \nu \tau+\sum_{n=1}^{\infty} c_{n}(\tau) \theta_{1}^{n} \\
& \theta_{s}(\tau)=\operatorname{sen} \nu \tau+\sum_{n=1}^{\infty} s_{n}(\tau) \theta_{1}^{n}
\end{aligned}
$$

onde $c_{n}(\tau)$ e $s_{n}(\tau)$ são funções a serem determinadas de modo a satisfazer a equação (7). Sendo assim, a solução geral da equação de movimento seria uma combinação linear das duas séries acima

$$
\theta(\tau)=A \theta_{c}(\tau)+B \theta_{s}(\tau)
$$

que também apresenta a propriedade de reduzir-se à solução de um oscilador harmônico simples caso $\theta_{1} \rightarrow 0$, uma combinação linear de seno e cosseno, ambos com a frequência natural do pêndulo $(\nu)$. A partir de agora, com a finalidade de simplificar a exposição do processo construtivo das soluções propostas, apenas a série 21) será desenvolvida.

Substituindo (21) e 20 em (7) e agrupando potências iguais de $\theta_{1}$ obtém-se:

$$
\begin{gathered}
\left(c_{1}^{\prime \prime}+\nu^{2} c_{1}+\alpha_{1} \cos \nu \tau+2 \cos 2 \tau \cos \nu \tau\right) \theta_{1} \\
+\sum_{n=2}^{\infty}\left(c_{n}^{\prime \prime}+\nu^{2} c_{n}+\alpha_{n} \cos \nu \tau+2 c_{n-1} \cos 2 \tau\right. \\
\left.+\sum_{m=1}^{n-1} \alpha_{m} c_{n-m}\right) \theta_{1}^{n}=0
\end{gathered}
$$

onde a notação $\beta^{\prime}$ está, aqui, indicando a derivada da grandeza $\beta$ em relação ao tempo adimensional $\tau$, ou seja, $\beta^{\prime}=\frac{d \beta}{d \tau}$. 
Uma vez que o parâmetro $\theta_{1}$ é arbitrário, a equação 24) será válida somente se cada coeficiente da série de potências for nulo. Essa condição, acrescida da exigência de que o coeficiente frente ao cosseno na série solução, equação 21, seja unitário ${ }^{2}$ permite determinar univocamente os $\alpha_{n}$ 's e $c_{n}$ 's que, por sua vez, são necessários para construir a solução final.

Isto posto, o coeficiente multiplicativo de $\theta_{1}^{1}$ fornece a seguinte equação diferencial

$$
c_{1}^{\prime \prime}+\nu^{2} c_{1}+\alpha_{1} \cos \nu \tau+\cos (\nu+2) \tau+\cos (\nu-2) \tau=0
$$

onde o produto $\cos 2 \tau \cos \nu \tau$ foi reescrito como uma soma de cossenos usando-se a identitdade trigonométrica

$$
\cos (m+n) x+\cos (m-n) x=2 \cos m x \cos n x .
$$

A solução da parte homogênea de 25 é:

$$
c_{1, H}(\tau)=C \cos \nu \tau+D \operatorname{sen} \nu \tau .
$$

Devido à convenção de tornarmos unitário o coeficiente que multiplica o $\cos \nu \tau$ na solução geral para $\theta$, somos obrigados a anular o coeficiente $C$. A solução em sen $\nu \tau$ é levada em consideração pela série $\theta_{s}(\tau)$, de modo que anula-se também o coeficiente $D$. Este procedimento tornará $c_{1}(\tau)$ igual à solução particular da equação $(25) \mathrm{e}$ se repetirá na obtenção de $c_{2}$ (e de todo $c_{n}$ ), de modo que posteriormente será omitida a apresentação da solução da parte homogênea da função $c_{2}(\tau)$.

É proposta a seguinte solução particular 11]:

$c_{1, P}(\tau)=A_{1} \tau \operatorname{sen} \nu \tau+B_{1} \cos (\nu+2) \tau+C_{1} \cos (\nu-2) \tau$

onde a substituição direta desta proposta na equação 25) determina os valores das constantes como $A_{1}=$ $-\frac{\alpha_{1}}{2 \nu}, B_{1}=\frac{1}{4(\nu+1)}$ e $C_{1}=-\frac{1}{4(\nu-1)}$. Seja notado o surgimento das seguintes restrições sobre o valor da frequência natural de oscilação: $\nu \neq 0, \pm 1$.

Para que a função $c(\tau)$ seja limitada e oscilante, a constante $A_{1}$ presente em $c_{1, P}$ deverá ser nula. Esse requisito é respeitado se fizermos $\alpha_{1}=0$, o que acaba por determinar o valor desta constante na série para $\theta_{0}$, equação 20 .

Com isso, a ordem 1 em $\theta_{1}$ nas séries dadas pelas equações 20 e 21) é explicitada pelos seguintes resultados:

$$
\begin{aligned}
\alpha_{1}= & 0 \\
c_{1}(\tau)= & \frac{1}{4}\left\{\frac{1}{\nu+1} \cos (\nu+2) \tau\right. \\
& \left.\quad-\frac{1}{\nu-1} \cos (\nu-2) \tau\right\} .
\end{aligned}
$$

Por sua vez, o coeficiente quadrático em $\theta_{1}$ na série (24) gera a seguinte equação diferencial

$$
c_{2}^{\prime \prime}+\nu^{2} c_{2}+\alpha_{2} \cos \nu \tau+\alpha_{1} c_{1}+2 c_{1} \cos 2 \tau=0 .
$$

${ }^{2}$ Essa exigência é arbitrária e não gera perda de generalidade na solução construída, uma vez que as equações diferenciais que estão sendo resolvidas são equações lineares.
Substituindo na equação acima os valores já conhecidos de $c_{1}$ e $\alpha_{1}$ e, então, reescrevendo-a com os produtos de cossenos sendo trocados por somas, por meio do uso da identidade (26), obtém-se

$$
\begin{aligned}
c_{2}^{\prime \prime}+\nu^{2} c_{2}= & -\left[\alpha_{2}+\frac{1}{4(\nu+1)}-\frac{1}{4(\nu-1)}\right] \cos \nu \tau \\
& -\frac{1}{4(\nu+1)} \cos (\nu+4) \tau \\
& +\frac{1}{4(\nu-1)} \cos (\nu-4) \tau
\end{aligned}
$$

Seguindo a mesma linha de raciocínio que permitiu determinar o coeficiente $\alpha_{1}$ e a função $c_{1}(\tau)$, chega-se nos seguintes resultados para $\alpha_{2}$ e $c_{2}(\tau)$

$$
\begin{aligned}
\alpha_{2}= & \frac{1}{2(\nu-1)(\nu+1)} \\
c_{2}(\tau)= & \frac{1}{32}\left[\frac{1}{(\nu+1)(\nu+2)} \cos (\nu+4) \tau\right. \\
& \left.\quad+\frac{1}{(\nu-1)(\nu-2)} \cos (\nu-4) \tau\right]
\end{aligned}
$$

com o acréscimo das seguintes restrições sobre o valor da frequência natural do oscilador harmônico $\nu \neq \pm 2$.

Com esse procedimento sendo seguido ordem a ordem nas potências de $\theta_{1}$ da série (24) determinam-se mais e mais constantes $\alpha_{n}$ e funções $c_{n}(\tau)$, tornando a descrição do movimento do pêndulo no entorno da posição invertida, dada pela função $\theta(\tau)$, cada vez mais próxima daquela obtida numericamente.

Tomando o coeficiente $\theta_{1} \ll 1$, que implica em termos a amplitude de oscilação do pivô muito menor que a distância deste até o centro de massa $(a \ll l)$, é possível truncar as séries (20) e (21) já no termo quadrático em $\theta_{1}$ e obter a seguinte solução aproximada, em cossenos, para a equação (7)

$$
\begin{aligned}
\theta_{c}(\tau) \cong & \cos \nu \tau \\
+ & \frac{1}{4} \theta_{1}\left[\frac{1}{\nu+1} \cos (\nu+2) \tau\right. \\
& \left.\quad-\frac{1}{\nu-1} \cos (\nu-2) \tau\right] \\
+ & \frac{1}{32} \theta_{1}^{2}\left[\frac{1}{(\nu+1)(\nu+2)} \cos (\nu+4) \tau\right. \\
& \left.+\frac{1}{(\nu-1)(\nu-2)} \cos (\nu-4) \tau\right] .
\end{aligned}
$$

sendo $\theta_{0}\left(\nu, \theta_{1}\right)$ dado por

$$
\theta_{0} \cong \nu^{2}+\frac{1}{2(\nu-1)(\nu+1)} \theta_{1}^{2}
$$

A solução correspondente em senos, $\theta_{s}(\tau)$ dada pela série (22), é construída de maneira análoga a apresentada para determinar a solução em cossenos e é dada por, até 
ordem 2 em $\theta_{1}$ :

$$
\begin{aligned}
\theta_{s}(\tau) \cong & \operatorname{sen} \nu \tau \\
+ & \frac{1}{4} \theta_{1}\left[\frac{1}{\nu+1} \operatorname{sen}(\nu+2) \tau\right. \\
& \left.\quad-\frac{1}{\nu-1} \operatorname{sen}(\nu-2) \tau\right] \\
+ & \frac{1}{32} \theta_{1}^{2}\left[\frac{1}{(\nu+1)(\nu+2)} \operatorname{sen}(\nu+4) \tau\right. \\
& \left.+\frac{1}{(\nu-1)(\nu-2)} \operatorname{sen}(\nu-4) \tau\right]
\end{aligned}
$$

De acordo com a referência [10 as funções $\theta_{s}(\tau)$ e $\theta_{c}(\tau)$ são denominadas seno elíptico e cosseno elíptico de ordem $\nu$, respectivamente, e são abreviadas por $\operatorname{se}_{\nu}\left(\tau, \theta_{1}\right)$ e $\operatorname{ce}_{\nu}\left(\tau, \theta_{1}\right)$. Na seção 2.16 daquela referência é citado que estas funções serão periódicas se $\nu$ for racional e não periódicas (mas ainda limitadas) caso $\nu$ seja irracional.

O processo construtivo adotado para determinar as funções $\theta_{c}(\tau)$ e $\theta_{s}(\tau)$ impõe que $\nu$ não poderá ser inteiro, caso contrário haverá singularidade na solução. Desse modo sabe-se que os casos em que $\nu$ seja inteiro deverão ser tratados à parte com este métodd ${ }^{3}$

É importante notar que não se mencionou nada a respeito do sinal dos parâmetros $\theta_{0}$ e $\theta_{1}$ durante a dedução, de modo que a definição de $\theta_{0}$ com sinal negativo não é empecilho nem limitação sobre os resultados apresentados.

Comentários e demonstrações a respeito da ortogonalidade de $\mathrm{Se}_{\nu}$ e ce ${ }_{\nu}$, a maneira mais conveniente de normalizar e representar em série estas funções de modo a permitir demonstrações de convergência, quando a solução aqui desenvolvida é válida (qual região de parâmetros), estabilidade dessas soluções, entre tantos outros assuntos específicos sobre estas funções, todos são encontrados e bem tratados na referência [10]. Todo esse rigor matemático é aqui deixado de lado por fugir completamente ao escopo da exposição proposta neste texto.

Até aqui as soluções apresentadas estão sob a restrição de que, dado um $\theta_{1}$ e uma 'frequência natural' $\nu$, o parâmetro $\theta_{0}$ seja exatamente aquele dado pela equação (27), este consistindo no modo de determinarmos os parâmetros $a$ e $\omega$ que fornecem a inversão do pêndulo, caso se deseje construir um. Daqui adiante, ao invés de serem tratados os parâmetros $\theta_{0}$ e $\theta_{1}$ serão novamente tratados diretamente os parâmetros originais, amplitude de forçamento $a$ e frequência de forçamento $\omega$, o que facilitará a interpretação dos resultados desta abordagem.

Substituindo as definições de $\theta_{0}$ e $\theta_{1}$, equações (5) e (6) respectivamente, na equação (27), pode-se escrever $\nu$

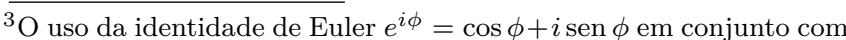
algumas identidades trigonométricas apropriadas permite relacionar as soluções (8) e (23), justificando o uso da série (8) como ansatz em 1 .
}

diretamente em função de $a$ e $\omega$

$$
\begin{aligned}
& \nu^{2}= {\left[\frac{1}{2}-2\left(\frac{\omega_{0}}{\omega}\right)^{2}\right] } \\
& \pm \sqrt{\left[\frac{1}{2}+2\left(\frac{\omega_{0}}{\omega}\right)^{2}\right]^{2}-2\left(\frac{m l^{2}}{I}\right)^{2}\left(\frac{a}{l}\right)^{2}}
\end{aligned}
$$

A soma e a subtração aqui presentes referem-se a uma frequência natural de oscilação superior à outra, sendo a maior frequência (sinal positivo) associada a oscilação do pêndulo no entorno da posição ordinária e a menor frequência (sinal negativo) associada a oscilação do pêndulo no entorno da posição invertida.

Para que a solução $\theta(\tau)$ seja oscilatória, conforme ocorre experimentalmente, a frequência $\nu$ deverá ser real, o que implica em termos $\nu^{2}>0$. Esta imposição implica na seguinte desigualdade (limite inferior) a ser obedecida para o parâmetro $\frac{a}{l}$, para um dado $\frac{\omega_{0}}{\omega}$

$$
\frac{a}{l}>\sqrt{2} \frac{I}{m l^{2}} \frac{\omega_{0}}{\omega} .
$$

Ainda é necessário impor que o termo dentro da raiz na equação que fornece o $\nu^{2}$ seja positivo. Esta imposição nos fornece um limite superior para o parâmetro $\frac{a}{l}$, novamente para um dado $\frac{\omega_{0}}{\omega}$

$$
\frac{a}{l}<\sqrt{2} \frac{I}{m l^{2}}\left[\frac{1}{4}+\left(\frac{\omega_{0}}{\omega}\right)^{2}\right] .
$$

Desse modo a imposição sobre a frequência $\nu$ ser real fornece os limites superior e inferior do parâmetro $\frac{a}{l}$ para um dado $\frac{\omega_{0}}{\omega}$. Isso, por sua vez, é a própria região de parâmetros que fornece estabilidade invertida do pêndulo, como era procurado. Agrupando resultados desta abordagem, escreve-se:

$$
\sqrt{2} \frac{I}{m l^{2}} \frac{\omega_{0}}{\omega}<\frac{a}{l}<\sqrt{2} \frac{I}{m l^{2}}\left[\frac{1}{4}+\left(\frac{\omega_{0}}{\omega}\right)^{2}\right] .
$$

Embora haja maior simplicidade no tratamento dado por Butikov [2] em relação ao aqui exposto, ganha-se um limite superior para a razão $\frac{a}{l}$, que lá não é encontrado. Por outro lado o truncamento da série que fornece $\theta_{0}$ tem o preço de prever um limite superior diferente daquele encontrado por Phelps-Hunter [1], cujo tratamento matemático é mais rigoroso que o exposto aqui [comparar inequações [12] e [28]]. Por fim, o trabalho do Butikov 2 tem previsões não restritas a pequenas oscilações no entorno da posição invertida enquanto Phelps-Hunter [1] e o apresentado aqui possuem esta restrição.

\section{Resultados numéricos}

Uma sequência de simulações numéricas do pêndulo com pivô verticalmente forçado foi efetuada com o objetivo de testar os resultados analíticos apresentados na Seção 
3 O procedimento adotado foi o seguinte: para testar se a condição dada na equação 28 é verdadeira, foi tomada a equação de movimento adimensional exata para o pêndulo (com ângulo contado da posição invertida no sentido horário)

$$
\frac{d^{2} \theta}{d \tau^{2}}+\left(-4 \frac{\omega_{0}^{2}}{\omega^{2}}+4 \frac{m l^{2}}{I} \frac{a}{l} \cos 2 \tau\right) \operatorname{sen} \theta=0
$$

e várias 'situações diferentes' foram simuladas usando-se o método de Runge-Kutta de quarta ordem (RK4) [12]. Essas 'situações diferentes' referem-se a uma varredura do espaço de parâmetros $\left(\frac{a}{l}, \frac{\omega_{0}}{\omega}\right)$ juntamente com uma varredura no espaço de condições iniciais $(\theta(0), \dot{\theta}(0))$ a fim de explicitar o quão robusta é uma combinação de parâmetros de forçamento na produção de estabilidade invertida. Lembrando que a teoria desenvolvida na seção anterior é válida para pequenas oscilações em torno da vertical, a varredura nas condições iniciais foi feita respeitando essa limitação. Ao todo foram testadas 100 condições iniciais distintas (grade 10x10 no espaço de condições iniciais) pertencentes ao intervalo $[0,0.1] \mathrm{em}$ ambas as constantes iniciais $(\theta(0), \dot{\theta}(0))$. Para cada uma dessas condições iniciais o espaço de parâmetros de forçamento $\left(\frac{a}{l}, \frac{\omega_{0}}{\omega}\right)$ foi varrido sistematicamente tomando-se valores de ambos no intervalo $[0,1]$ (grade 10x10 no espaço de parâmetros) e registrando-se em um arquivo somente aqueles pares de parâmetros, dada uma condição inicial, que geravam estabilidade invertida. O critério de estabilidade adotado foi a obtenção de um $\theta$ médio menor que 0.1 em aproximadamente 300 períodos de oscilação do pivô, que corresponderia a 10 ou mais oscilações do pêndulo no caso de ser gerada establidade invertida.

No arquivo final gerado pelo procedimento anteriormente descrito havia, para cada condição inicial testada, um certo número de pares de parâmetros de forçamento que produzia estabilidade. Tal número de pares de parâmetros de forçamento que produziam a estabilidade variou entre 5 e 14, notando-se que quanto mais robusta a condição inicial (maior velocidade angular e ângulo iniciais), menor era o número de parâmetros que dava estabilidade.

Foram tomados então os dois extremos de condições iniciais, os que forneciam $5(\phi(0)=0,1$ e $\dot{\phi}(0)=0,1)$ e $14(\phi(0)=0,06$ e $\dot{\phi}(0)=0,01)$ pares de parâmetros $\left(\frac{a}{l}, \frac{\omega_{0}}{\omega}\right)$ produzindo estabilidade invertida e, novamente, o espaço de parâmetros foi varrido no mesmo intervalo anterior. Desta vez foram tomadas 10000 combinações diferentes destes pares de parâmetros (grade 100x100) e registrados aqueles que forneciam estabilidade (sob o mesmo critério anterior de estabilidade). As Figuras 3 e 4 apresentam os resultados numéricos assim obtidos para os pares de parâmetros que produzem estabilidade invertida ao pêndulo (círculos vazios). Uma comparação direta com a previsão analítica da região de establidade (linhas sólidas) dada pela equação 28 pode ser visualizada nestas figuras. Deve-se notar que a previsão da

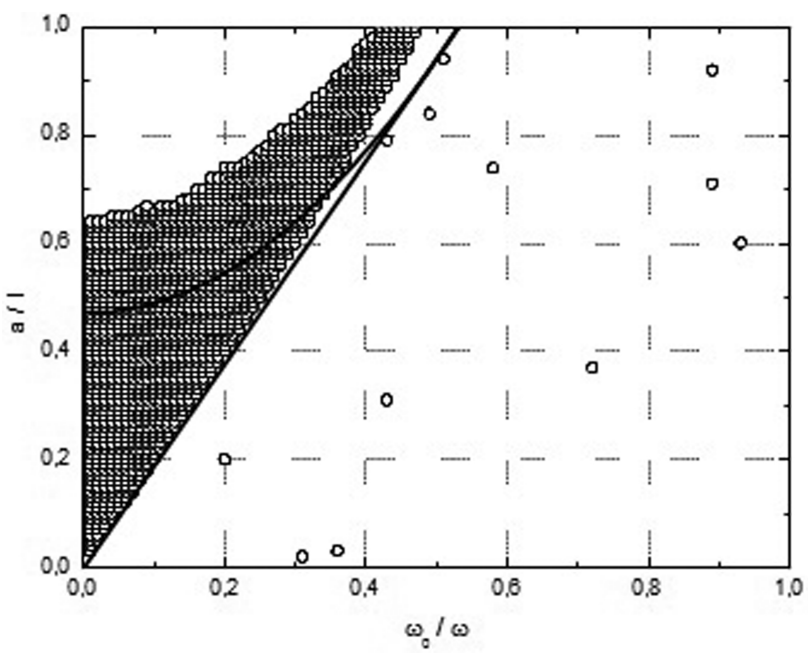

Figura 3: Cada círculo aberto corresponde a uma combinação de parâmetros $\left(a / l, \omega_{0} / \omega\right)$ que produz dinâmica invertida estável do pêndulo com pivô verticalmente forçado para condição inicial $\phi(0)=0,01$ e $\dot{\phi}(0)=0,06$. As linhas sólidas indicam os limites de valores possíveis para os parâmetros $\left(a / l, \omega_{0} / \omega\right)$ que gerarão estabilidade invertida, conforme indicados pela equação (28).

região de parâmetros produtores de estabilidade está subestimada em comparação com os resultados numéricos, consequência direta do truncamento no termo de ordem $\theta_{1}^{2}$ na série solução proposta.

Os círculos vazios "espalhados" da região de estabilidade nas Figuras 3 e 4 correspondem a trajetórias instáveis que, devido a alternância entre oscilação e rotação, acabaram por obedecer o critério de estabilidade adotado.

Por fim, uma simulação numérica do pêndulo invertido é apresentada na Figura 5 para a condição inicial $(\phi(0)=$

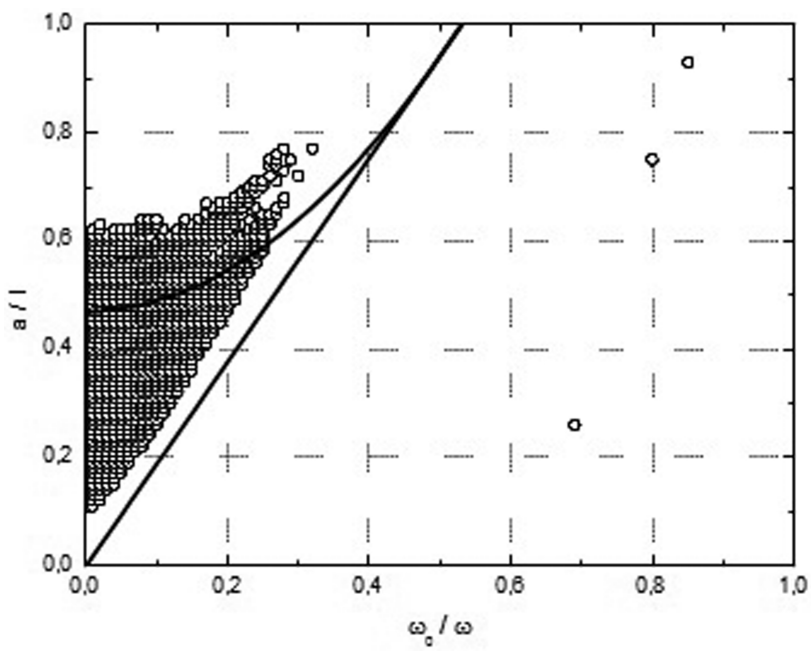

Figura 4: Cada círculo aberto corresponde a uma combinação de parâmetros $\left(a / l, \omega_{0} / \omega\right)$ que produz dinâmica invertida estável do pêndulo com pivô verticalmente forçado para condição inicial $\phi(0)=0,1$ e $\dot{\phi}(0)=0,1$. As linhas sólidas indicam os limites de valores possíveis para os parâmetros $\left(a / l, \omega_{0} / \omega\right)$ que gerarão estabilidade invertida, conforme indicados pela equação (28). 


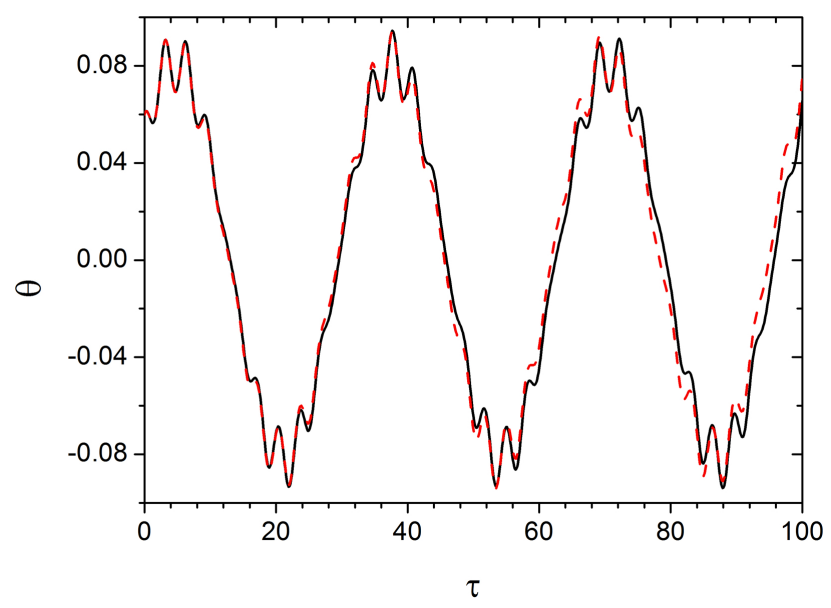

Figura 5: Condições iniciais: $\phi(0)=0,06$ e $\dot{\phi}(0)=0,01$. A linha sólida (preta) corresponde ao resultado numérico e a linha tracejada (vermelha) corresponde ao resultado analítico.

$0,06$ e $\dot{\phi}(0)=0,01)$ e para os parâmetros de oscilação do pivô dados por $\left(\frac{a}{l}=\frac{1}{5}, \frac{\omega_{0}}{\omega}=\frac{1}{20}\right)$, com a finalidade de comparar a solução analítica $\theta(\tau)$, equação $(23)$, com o resultado numérico. É possível ver que, embora a série infinita proposta como solução tenha sido truncada já no termo de ordem 2, há razoável proximidade entre a curva simulada e a analítica.

\section{Considerações finais}

A análise desenvolvida ao longo deste trabalho fixouse unicamente na descrição das trajetórias invertidas estáveis do pêndulo com pivô verticalmente forçado, sob a restrição de pequenas amplitudes de forçamento $(a \ll l)$ e grandes frequências de forçamento $\left(\omega \gg \omega_{0}\right)$. Entretanto, cabe observar que um pêndulo forçado sem as restrições aqui impostas pode fornecer muitas dinâmicas distintas: caóticas, de ressonância paramétrica, de rotação pura, de rotação alternada com oscilação, entre muitas outras. Trata-se de um sistema valioso para introduzir métodos matemáticos no nível superior.

Ao comparar as três abordagens dadas ao fenômeno da inversão do pêndulo pela excitação vertical de seu pivô e notar que o tratamento dado por Phelps-Hunter [1] tem grande elegância matemática porém pouca clareza a respeito da razão física que leva a inversão, que o tratamento dado por Butikov 2 fornece clareza sobre a razão física que gera a inversão às custas de uma argumentação matemática eurística, e que o tratamento dado aqui tem simplicidade matemática com uma argumentação também eurística, percebe-se que abordagens diferentes dadas a um único sistema fornecem uma oportunidade de amadurecimento profissional, técnico e intuitivo, dificilmente encontrada nos tratamentos comuns obtidos durante a formação inicial em física.

Por fim, os resultados das Figuras 3, 4, e 5, juntamente com aqueles das referências de comparação, PhelpsHunter 1] e Butikov 2], servem para solidificar o fato de que o uso de aproximações não deve ser desvalorizado, mesmo que estas limitem os resultados.

\section{Agradecimentos}

TCL tem profunda gratidão à paciente orientação do Prof. Dr. Frederico Firmo de Souza Cruz durante os anos de iniciação científica, mestrado e doutorado. Esta orientação em conjunto com longas e agradáveis discussões renderam muitos aprendizados que os livros não podem fornecer.

\section{Referências}

[1] F.M. Phelps III and J.H. Hunter Jr., American Journal of Physics 33, 285 (1965)

[2] E.I. Butikov, American Journal of Physics 69, 755 (2001)

[3] G. J. Mata, E. Pestana, European Journal of Physics 25, 717 (2004)

[4] H.P. Kalmus, American Journal of Physics 38, 874 (1970)

[5] L. Blitzer, American Journal of Physics 33, 1076 (1965)

[6] E.D. Yorke, American Journal of Physics 46, 285 (1978)

[7] D.J. Ness, American Journal of Physics 35, 964 (1967)

[8] A.B. Pippard, European Journal of Physics 8, 203 (1987)

[9] R. Eisberg, R. Resnick, Física Quântica: átomos, moléculas, sólidos, núcleos e partículas, (Elsevier Editora Ltda, Rio de Janeiro, 1979), cap. 12.

[10] N.W. McLachlan, Theory and application of Mathieu functions, (Dover Publications, Inc., New York, 1964).

[11] L. Leithold, O cálculo com geometria analítica, (Editora Harbra Ltda, São Paulo, 1994), 3ª ed, v.2, cap. 20.

[12] P.L. DeVries, A first course in computational physics, (John Wiley and Sons, Massachusetts, 1994), cap. 5. 